\title{
An Aeromonas salmonicida type IV pilin is required for virulence in rainbow trout Oncorhynchus mykiss
}

\author{
Cynthia L. Masada ${ }^{1}$, Scott E. LaPatra ${ }^{2}$, Andrew W. Morton ${ }^{2}$, Mark S. Strom ${ }^{1, *}$ \\ ${ }^{1}$ Northwest Fisheries Science Center, National Marine Fisheries Service, National Oceanic and Atmospheric Administration, \\ United States Department of Commerce, 2725 Montlake Boulevard East, Seattle, Washington 98112, USA \\ ${ }^{2}$ Clear Springs Foods, PO Box 712, Buhl, Idaho 83316, USA
}

\begin{abstract}
Aeromonas salmonicida expresses a large number of proven and suspected virulence factors including bacterial surface proteins, extracellular degradative enzymes, and toxins. We report the isolation and characterization of a 4-gene cluster, tap $A B C D$, from virulent A. salmonicida A450 that encodes proteins homologous to components required for type IV pilus biogenesis. One gene, $\operatorname{tap} A$, encodes a protein with high homology to type IV pilus subunit proteins from many Gramnegative bacterial pathogens, including Aeromonas hydrophila, Pseudomonas aeruginosa, and Vibrio vulnificus. A survey of $A$. salmonicida isolates from a variety of sources shows that the $\operatorname{tap} A$ gene is as ubiquitous in this species as it is in other members of the Aeromonads. Immunoblotting experiments demonstrate that it is expressed in vitro and is antigenically conserved among the A. salmonicida strains tested. A mutant A. salmonicida strain defective in expression of TapA was constructed by allelic exchange and found to be slightly less pathogenic for juvenile Oncorhynchus mykiss (rainbow trout) than wild type when delivered by intraperitoneal injection. In addition, fish initially challenged with a high dose of wild type were slightly more resistant to rechallenge with wild type than those initially challenged with the $\operatorname{tap} A$ mutant strain, suggesting that presence of TapA contributes to immunity. Two of the other three genes identified, $\operatorname{tap} B$ and $\operatorname{tap} C$, encode proteins with homology to factors known to be required for type IV pilus assembly in $P$. aeruginosa, but in an as yet unidentified manner. TapB is a member of the ABC-transporter family of proteins that contain characteristic nucleotide-binding regions, and which may provide energy for type IV pilus assembly through the hydrolysis of ATP. TapC homologs are integral cytoplasmic membrane proteins that may play a role in pilus anchoring or initiation of assembly. The fourth gene, $t a p D$, encodes a product that shares homology with a family of proteins with a known biochemical function, namely, the type IV prepilin leader peptidases. These bifunctional enzymes proteolytically cleave the leader peptide from the pilin precursor (prepilin) and then $N$-methylate the newly exposed $N$-terminal amino acid prior to assembly of the subunits into the pilus structure. We demonstrate that $A$. salmonicida TapD is able to restore type IV pilus assembly and type II secretion in a $P$. aeruginosa strain carrying a mutation in its type IV peptidase gene, suggesting that it plays the same role in A. salmonicida.
\end{abstract}

KEY WORDS: Virulence · Type IV pilin · Type II protein secretion

Resale or republication not permitted without written consent of the publisher

\section{INTRODUCTION}

Aeromonas salmonicida is an opportunistic and ubiquitous fish pathogen that causes the disease furunculosis in salmonids. The disease can be acute or chronic. In the former case fish die from a massive toxemia with

*Corresponding author. E-mail: mark.strom@noaa.gov few external signs of disease, while chronic furunculosis results in localized hemorrhaging and necrosis in the gills, gut, and muscle, petechiae at fin bases, and sometimes gross swellings (furuncles) and extensive hemorrhaging from gills. A number of individual extracellularly secreted proteins (often called ECPs for extracellular proteins) and bacterial surface factors have been implicated in the virulence and pathogenesis of 
A. salmonicida. These include proteases (Sakai 1985), hemolysins and cytolysins (Hirono \& Aoki 1993, Ellis 1997), a phospholipase (Buckley et al. 1982), a paracrystalline surface array protein (S-layer) (Trust 1993), and an extracellular polysaccharide capsule (Merino et al. 1996).

In recent studies we and colleagues have described the isolation of genes encoding factors involved in the biogenesis of type IV pili and type II extracellular secretion of proteins from Aeromonas hydrophila (Pepe et al. 1996, Barnett et al. 1997, Barnett \& Kirov 1999, Kirov et al. 2000). Type IV pili are present on the surface of many Gram-negative pathogens and have been shown to be important virulence determinants (Strom \& Lory 1993). Type IV pili act primarily as adhesins by promoting adherence and colonization of epithelial cells. However, they have also been implicated in other functions that may contribute to in vivo growth and pathogenesis, such as motility, biofilm formation, DNA uptake, cell signaling, and as receptors for bacteriophages. The type II mechanism of extracellular protein secretion, also called the main terminal branch of the general secretory pathway (Pugsley 1993, Pugsley et al. 1997), is related to biogenesis of type IV pili in that many proteins of each system have homologs in the other (Strom \& Lory 1993, Nunn 1999). For example, the protein subunit of type IV pili, pilin, is homologous to several membrane proteins that form part of the type II secretion machinery. The pilins and the type II pathway 'pseudopilins' are first synthesized in precursor form with a specialized amino terminal leader peptide that must be proteolytically cleaved by a type IV leader peptidase prior to assembly of the pilus or the type II secretory complex. The type IV leader peptidase is an enzyme distinct from the leader peptidase I of the Sec pathway. In many Gram-negative pathogens that possess both type IV pili and secrete proteins via the type II pathway, including A. hydrophila, Vibrio vulnificus, and Pseudomonas aeruginosa, it has been shown that a single type IV leader peptidase is required for type IV pilus biogenesis and type II secretion (Strom et al. 1991, 1993, Pepe et al. 1996, Paranjpye et al. 1998). Mutations in the type IV peptidase gene caused a pleiotropic defect with the mutants unable to express pili or secrete many extracellular proteins, and can be attenuated for virulence (Paranjpye et al. 1998, Fullner \& Mekalanos 1999).

In this study, we report the cloning and characterization of a type IV pilus biogenesis gene cluster from Aeromonas salmonicida. The cluster contains genes encoding a typical type IV leader peptidase and a type IV pilin. The type IV pilin and type IV leader peptidase are expressed under in vitro conditions. The role of the type IV pilin in virulence in rainbow trout Oncorhynchus mykiss is also assessed.

\section{MATERIALS AND METHODS}

Bacterial strains, plasmids, and growth conditions. The bacterial strains and plasmids used in this study are listed in Table 1. Aeromonas salmonicida strains were grown at $22^{\circ} \mathrm{C}$ in trypticase soy (TS) medium, while Escherichia coli and Pseudomonas aeruginosa strains were grown at $37^{\circ} \mathrm{C}$ in Luria-Bertani (LB) medium. The following antibiotic concentrations (in $\mu \mathrm{g}$ $\mathrm{ml}^{-1}$ ) were used for A. salmonicida: ampicillin, 50; kanamycin, 25; nalidixic acid, 5. For E. coli, the concentrations were as follows: ampicillin, 150; carbenicillin, 50; chloramphenicol, 20; gentamicin, 10 or 30; kanamycin, 50. For $P$. aeruginosa, the concentrations were as follows: carbenicillin, 200; streptomycin, 300; tetracycline, 200. Isopropyl thio- $\beta$-D-thiogalactoside (IPTG) was used at a final concentration of $0.5 \mathrm{mM}$.

DNA and RNA methods. Small-scale plasmid preparations were performed using the alkaline lysis protocol (Birnboim \& Doly 1979). For large-scale plasmid preparation, Qiagen midi-prep columns were used. Aeromonas salmonicida chromosomal DNA was extracted as described for Pseudomonas aeruginosa DNA (Strom \& Lory 1986). Restriction endonuclease digestion, ligation, transformation, and DNA electrophoresis were performed as described by Sambrook et al. (1989). Broad-host range plasmids were introduced into $A$. salmonicida and $P$. aeruginosa by conjugation from Escherichia coli S17-1גpir (Simon et al. 1983, Miller \& Mekalanos 1988). Northern blot hybridization by agarose-formaldehyde gel electrophoresis was performed as described by Sambrook et al. (1989). The Genius ${ }^{\mathrm{TM}}$ System non-radioactive detection kit (Boehringer Mannheim) was used for Southern and northern blot analyses with random-primed digoxigenin-labeled DNA probes.

Construction of expression plasmids. To construct an Aeromonas salmonicida tapA-overexpressing plasmid, a Hind III site was created upstream of the tapA coding region (AAGGAC changed to AAGCTT at positions 1167 to 1172 of the $A$. salmonicida tapAB sequence; GenBank accession number AF059248) and an XbaI site was created downstream of the coding region (GCTAGA changed to TCTAGA at positions 1631 to 1626 of the $A$. salmonicida tap $A B$ sequence) by PCR using AsalA-H3 (5'-CAG GAA CCA AGC TTA AAA GGA CAT TTG-3') and AsalA-Xba (5'-CCG CTA TTG GGT CTA GAG GTC ATG-3') as primers and pCP1198 as template. To construct an A. salmonicida tapD-overexpressing plasmid, a Hind III site was created upstream of the tapD coding region (CCTTTT changed to AAGCTT at positions 1346 to 1351 of the A. salmonicida tapCD sequence; GenBank accession number AF059249) and an XbaI site was created downstream of the coding region (TATACA changed 


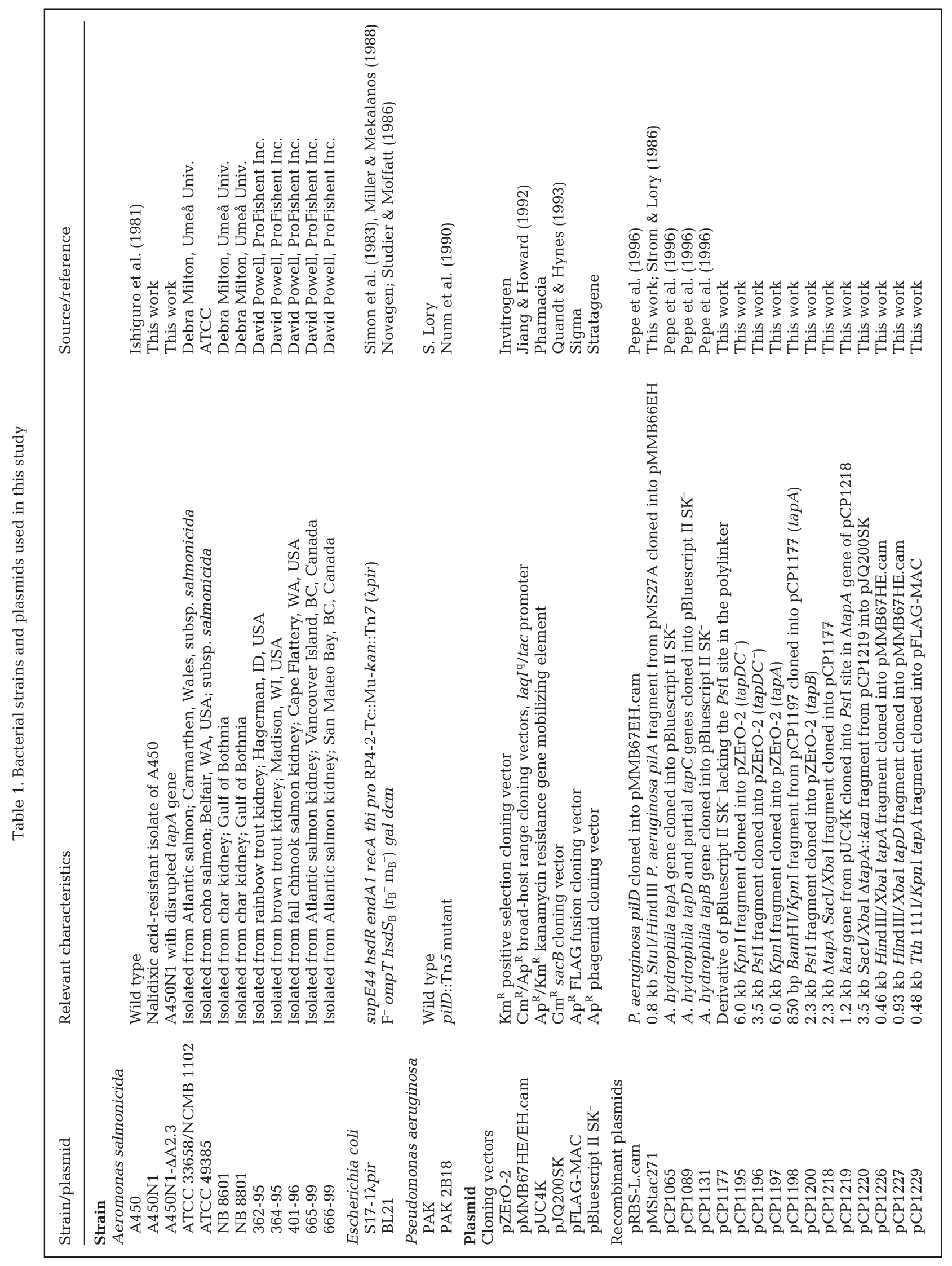


to TCTAGA at positions 2279 to 2274 of the A. salmonicida tapCD sequence) by PCR using AsalD-H3 (5'ACG GCA TGC AAG CTT CCC AAT TTC-3') and AsalD-Xba (5'-ATT GCA ACG ATC TAG ATG GGT CAG TG-3'). The PCR products containing tapA or $\operatorname{tapD}$ were then digested with HindIII and XbaI and ligated into pMMB67HE.cam (Jiang \& Howard 1992) digested with the same enzymes, generating pCP1226 and pCP1227, respectively. The structures of these plasmids were confirmed by sequencing. To construct a tapA-FLAG fusion plasmid, a Tth 111I site was created within the $\operatorname{tap} A$ coding region (TCAGGCTTT changed to GACAAAGTC at positions 1200 to 1208 of the A. salmonicida tapAB sequence) and a KpnI site was created downstream of the coding region (GGACTC changed to GGTACC at positions 1685 to 1680 of the $A$. salmonicida $\operatorname{tap} A B$ sequence) by PCR using F7V-AsalA (5'-TGA AGA AGC AAG ACA AAG TCA CCC TTA TTG-3') and C-Kpn-AsalA (5'-GTG AGT CGG TAC CAC TGA GCA G-3') as primers and pCP1196 as template. The PCR product containing $\operatorname{tap} A$ was then digested with Tth $111 \mathrm{I}$ and KpnI and ligated into pFLAG-MAC (Sigma) digested with the same enzymes, generating pCP1229. The cloning strategy was designed to fuse the FLAG peptide directly to the amino-terminus of the processed pilin protein, where the N-terminal amino acid, phenylalanine, is replaced by a valine. The construct and reading frame were confirmed by sequencing.

Hemolysis assay. Hemolytic activity was analyzed from the Pseudomonas aeruginosa pilD mutant strain PAK 2B18 containing either a type IV leader peptidase-producing construct (pRBS-L.cam or pCP1227) or the vector (pMMB67HE.cam) as follows. Each strain was grown overnight in $1.5 \mathrm{ml}$ LB containing carbenicillin and IPTG. Using a $1 \mu \mathrm{l}$ inoculating loop, a loopful of culture was carefully patched onto Columbia agar plates containing $5 \%(\mathrm{v} / \mathrm{v})$ rabbit erythrocytes. The plate was incubated overnight at $37^{\circ} \mathrm{C}$. A zone of clearing around the bacterial growth indicates secretion of hemolytic activity.

In vitro prepilin cleavage assay. In order to monitor cleavage activity of PilD and TapD, membrane fractions were prepared from the Pseudomonas aeruginosa pilD mutant strain PAK 2B18 containing either a type IV leader peptidase-producing construct (pRBSL.cam or pCP1227) or the vector (pMMB67HE.cam) as described previously (Pepe et al. 1996). Crude PilA prepilin substrate was prepared from the $P$. aeruginosa pilD mutant strain PAK B30 containing a PilA-producing construct (pMStac271; Strom \& Lory 1986) using the same method. The prepilin cleavage assays were performed as described by Strom et al. (1993). Standard $16 \%$ sodium dodecyl sulphate polyacrylamide gel electrophoresis (SDS-PAGE, Laemmli 1970) was used to analyze the reaction products $(6.25 \mu \mathrm{l}$ of each sample).

Construction of an Aeromonas salmonicida tapA mutant. A mutation in $\operatorname{tap} A$ was constructed by deleting an internal region of $\operatorname{tap} A$ then inserting a kanamycin-resistance cassette as a marker. The wild-type tapA gene in A. salmonicida A450N1 was replaced by allelic exchange using the plasmid pCP1220, which contains a mutated copy of $\operatorname{tap} A$ where $\sim 60 \%$ of the internal sequence has been removed and replaced with a kanamycin-resistance cassette. To construct pCP1220, the PstI site in the pBluescriptII SK-polylinker was first eliminated using the Klenow fragment of DNA polymerase followed by religation, resulting in $\mathrm{pCP} 1177$. Second, the internal deletion of tapA was generated within the tapA gene on pCP1197 by PCR. The PCR strategy was designed to create a PstI site at the deletion junction within $\operatorname{tap} A$ for subsequent cloning purposes and was carried out as follows. To generate the $5^{\prime}$ portion of the $\operatorname{tap} A$ gene, a SacI site was created upstream of the $\operatorname{tap} A$ coding region (AAGCTC changed to GAGCTC at positions 66 to 71 of the A. salmonicida tap $A B$ sequence) and a PstI site was created $\sim 40$ bases into the $\operatorname{tap} A$ coding region (CATCAG changed to CTGCAG at positions 1226 to 1221 of the $A$. salmonicida $\operatorname{tap} A B$ sequence) by PCR using N-Sac-AsalA2 (5'-GCC CTC ACC GAG CTC GAT CAA C-3') and N-Pst-AsalA (5'-CCA CGA TCT GCA GTT CAA TAA GGG-3') as primers and pCP1197 as template. For the $3^{\prime}$ portion of the $\operatorname{tap} A$ gene, a PstI site was already present in the wild-type $\operatorname{tap} A$ sequence at position 1480. An XbaI site was created downstream of the tapA coding region (TATAGA changed to TCTAGA at positions 2637 to 2629 of the A. salmonicida tap $A B$ sequence). The 3 ' portion of the $\operatorname{tap} A$ gene was generated by PCR using C-Pst-AsalA (5'-ATG CGA TCA CTG CAG AAG GAG TTG C-3') and C-Xba-AsalA (5'-GAG GCC GGT CTA GAG AGA GAC CG-3') as primers and genomic DNA from $A$. salmonicida A450 as template. The resulting PCR products were digested with $S a c \mathrm{I} / P s t \mathrm{I}$ or $P s t \mathrm{I} / \mathrm{XbaI}$, then combined and ligated with $S a c I / X b a I-d i g e s t e d$ pCP1177, generating pCP1218. (The SacI/XbaI fragment contains an internal PstI site and is referred to as $\Delta \operatorname{tap} A$.) Third, the $1.2 \mathrm{~kb}$ kan cassette from pUC4K (Pharmacia) was excised as a PstI fragment and inserted into the PstI site (at the deletion junction) present within the $\Delta \operatorname{tap} A$ gene of pCP1218, resulting in pCP1219. pCP1220 was constructed by cloning the $\sim 3.5 \mathrm{~kb}$ SacI-XbaI $\Delta$ tapA::kan fragment from pCP1219 into SacI/XbaI-digested pJQ200SK (Quandt \& Hynes 1993). To construct the tapA mutant strain, pCP1220

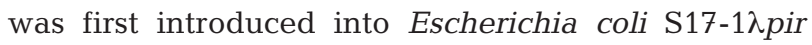
(Simon et al. 1983, Miller \& Mekalanos 1988) by transformation and then into A. salmonicida A450N1 by 
conjugation. Transconjugants were plated on TS agar containing nalidixic acid, kanamycin, and $5 \%(\mathrm{w} / \mathrm{v})$ sucrose to induce expression of the lethal $s a c B$ gene product (Gay et al. 1985). Growth in the presence of both sucrose and kanamycin requires recombination of the $\Delta \operatorname{tap} A:: k a n$ gene into the chromosome with subsequent loss of plasmid sequences. Gentamicin-sensitive candidates were examined by Southern blot analysis (see above) using a digoxigenin-labeled probe corresponding to a $404 \mathrm{bp} H i n c$ II-PstI fragment that represents most of the tapA gene (see Fig. 1). Several positive candidates were recovered, with A450N1- $\Delta$ A2.3 being chosen for further studies.

Purification of TapA-FLAG fusion protein and production of anti-TapA antiserum. To prepare antiserum against TapA from Aeromonas salmonicida A450, overnight cultures of Escherichia coli BL21 carrying pCP1229 were inoculated (1:100) into 3 flasks each containing $500 \mathrm{ml}$ of LB with carbenicillin and $0.4 \%(\mathrm{v} / \mathrm{v})$ glucose and grown at $37^{\circ} \mathrm{C}$ with shaking (200 rpm). When the $\mathrm{OD}_{600}$ reached $\sim 0.2$, IPTG was added and the cells were grown for $8 \mathrm{~h}$ at $22^{\circ} \mathrm{C}$ with shaking (100 rpm). The TapA-FLAG fusion protein was isolated from whole cell extracts and purified over an anti-FLAG M2 affinity gel column according to the manufacturer's protocols (FLAG E. coli Expression System; Sigma). The procedure described above was repeated several times until a total of $\sim 3.5 \mathrm{mg}$ of the TapA-FLAG fusion protein was generated. Antiserum was prepared in New Zealand White rabbits by R \& R Rabbitry Research Development. Briefly, 2 rabbits were each administered $\sim 0.5 \mathrm{mg}$ of the TapA-FLAG fusion protein in Freund's complete adjuvant. Five booster injections of $\sim 0.25 \mathrm{mg}$ fusion protein in Freund's incomplete adjuvant were given at Days 21, 42, 63, 84, and 105. Test bleeds were collected on Days 55 and 76, and $1 \%$ bleeds were collected on Days 97 and 118 . Serum from the Day 118 bleed of the rabbit showing the highest reactivity was used in this study. The antiserum was tested for TapA specificity by adsorption against an acetone powder preparation of A450N1$\Delta$ A2.3. Fig. 4, lane 5 ('Results') shows the anti-TapA antiserum reacting with the purified TapA-FLAG fusion protein.

Western blot analyses. Whole-cell lysates were prepared by spinning down $300 \mu \mathrm{l}$ of a TS broth overnight culture, then resuspending the pellet in $10 \mu \mathrm{l}$ water and adding $2.5 \mu \mathrm{l} 5 \times$ sample buffer $(0.3125 \mathrm{M}$ Tris- $\mathrm{HCl}$, pH $6.8 ; 50 \%$ (v/v) glycerol; $10 \%$ (w/v) SDS; $0.25 \%$ (v/v) 2-mercaptoethanol; $0.5 \%$ (w/v) bromophenol blue). For cell fractionations, osmotic shock was used to release periplasmic contents (Yim \& Villarejo 1992). Cytoplasmic and membrane fractions were extracted from the pellets that remained after osmotic shock as described (Manoil \& Beckwith 1986). For each of the fractionated samples, $20 \mu \mathrm{l}$ aliquots were mixed with $5 \mu \mathrm{l} 5 \times$ sample buffer and resolved by SDS-PAGE as described by Laemmli (1970) using discontinuous $16 \%$ acrylamide gels. The proteins were transferred to nitrocellulose (Towbin et al. 1979), incubated with anti-TapA polyclonal antiserum (see above), and visualized with goat anti-rabbit alkaline phosphatase conjugate (Promega).

Rainbow trout challenges. Wild-type and tapA mutant Aeromonas salmonicida strains, A450N1 and A450N1- $\Delta$ A2.3, respectively, were tested in rainbow trout as follows. Stock $1 \%(\mathrm{w} / \mathrm{v})$ bacterial suspensions were prepared in $0.5 \%(\mathrm{w} / \mathrm{v})$ tryptone after growth on TS agar plates. Triplicate tanks of rainbow trout (30 per tank; $\sim 5 \mathrm{~g}$ ) were inoculated intraperitoneally with $1: 100,1: 1000$, and 1:10 000 dilutions of the $1 \%$ stocks, or tryptone alone as a negative control. Mortality was followed for 3 wk and $\mathrm{LD}_{50}$ values were calculated (Reed \& Muench 1938). To test for any differences in protection against subsequent $A$. salmonicida challenge, the fish were rested for 3 additional weeks and then rechallenged with a 1:100 dilution of a $1 \%$ stock suspension of wild-type A. salmonicida strain A450N1. The relative percent survival (RPS; Amend 1981) was determined after following mortality for $3 \mathrm{wk}$.

\section{RESULTS}

\section{Isolation and sequence analysis of an Aeromonas salmonicida type IV pilus biogenesis gene cluster}

To determine whether Aeromonas salmonicida encodes genes required for type IV pilus biogenesis, chromosomal DNA from A. salmonicida A450 was analyzed by Southern blot hybridization (Genius $^{\mathrm{TM}}$ System, Boehringer Mannheim) using digoxigeninlabeled probes derived from the A. hydrophila tapA, tapB, tapC, and tapD genes (Fig. 1; Pepe et al. 1996). Homologous A. salmonicida sequence was detected with each of the A. hydrophila tap probes (data not shown).

Based on the restriction fragment lengths determined by Southern blot, a cloning strategy was designed to directly insert the putative Aeromonas salmonicida tap genes into a positive selection plasmid vector, pZErO-2 (Invitrogen). Several candidates were obtained and subsequently screened for the presence of tap gene sequence. Four plasmid candidates, pCP1195, pCP1196, pCP1197, and pCP1200, were found to contain DNA inserts that hybridized with tap gene probes (Fig. 1).

pCP1195 contains a 6 kb KpnI fragment, pCP1196 contains a $\sim 3.5 \mathrm{~kb}$ PstI fragment, pCP1197 contains a $\sim 6 \mathrm{~kb} \mathrm{KpnI} \mathrm{fragment} \mathrm{(distinct} \mathrm{from} \mathrm{the} \mathrm{KpnI} \mathrm{fragment}$ 


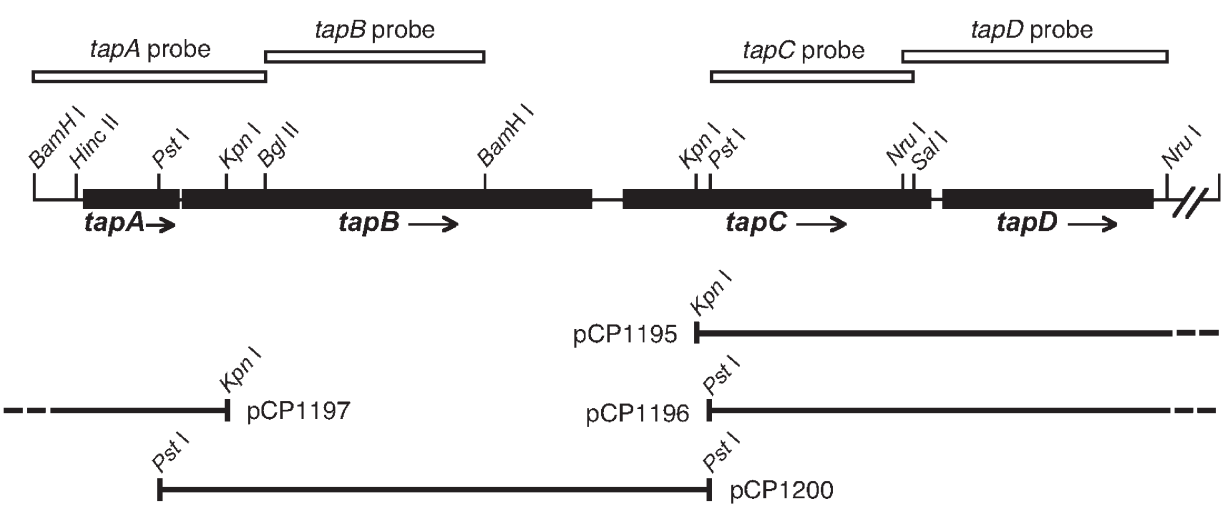

Fig. 1. Identification of type IV pilus biogenesis genes in Aeromonas salmonicida. Probes derived from $A$. hydrophila sequences used for Southern blotting $A$. salmonicida DNA are indicated above the restriction map of the A. salmonicida tap genes. Relevant restriction sites are shown. A. salmonicida tap fragments cloned into pZErO-2 to generate pCP1195, pCP1196, pCP1197, and pCP1200 are shown

in $\mathrm{pCP} 1195)$, and $\mathrm{pCP} 1200$ contains a 2.3 kb PstI fragment. All 4 plasmids were sequenced to determine if the cloned fragments encode genes homologous to the Aeromonas hydrophila tap genes. Sequence data indicate that the clones encompass 4 genes with high homology to $A$. hydrophila tap $A, \operatorname{tap} B, \operatorname{tap} C$, and tapD. The DNA sequences and encoded protein translations have been deposited in GenBank (accession nos. AF059248 and AF059249). A restriction map of the $A$. salmonicida tap gene cluster is shown in Fig. 1.

A BLAST search (Basic Local Alignment Search Tool; Altschul et al. 1997) of the GenBank database revealed that these Aeromonas salmonicida genes encode open reading frames (ORFs) that are highly homologous to previously characterized type IV pilins and associated pilus biogenesis proteins from several bacterial species, including A. hydrophila, Vibrio vulnificus, and Pseudomonas aeruginosa (Nunn et al. 1990, Pepe et al. 1996, Paranjpye et al. 1998). The A. salmonicida proteins share the highest level of homology with the corresponding A. hydrophila Tap proteins: TapA-62\% identity, TapB-98\% identity, TapC-97\% identity, and TapD-91\% identity (Fig. 2). These homologies allow assignment of functions to the A. salmonicida proteins. TapA is a typical type IV pilin subunit and will be discussed further below. TapB and TapC are type IV pilus biogenesis accessory proteins known to be required for assembly of the pilin subunits into pili (Nunn et al. 1990). While the precise role of these proteins in type IV pilus biogenesis is not known, TapB is a member of the ABC-transporter superfamily of proteins that bind nucleotides and hydrolyze ATP, providing energy for a variety of biochemical processes, and TapC is an integral cytoplasmic membrane protein that may be involved in pilus anchoring or initiation of assembly. A mutation in the ATP-binding domain of PilB, the TapB homolog in P. aeruginosa, results in a defect in pilus assembly in that organism (Turner et al. 1993). Likewise, inactivation of the TapC homolog PilC results in a similar pilus assembly defect (Nunn et al. 1990). TapD is a member of a family of bi- functional enzymes with endopeptidase and $N$-methyltransferase activites. These proteins are responsible for processing type IV prepilins and prepilin-like proteins into their mature forms (Nunn et al. 1990, Nunn \& Lory 1991, Strom et al. 1993).

Despite the high level of homology shared by type IV pilins, this homology is most prominent in the predominantly hydrophobic N-terminal domain of the polypeptide and does not extend into the region of greatest antigenicity near the carboxy-terminus (Strom \& Lory 1993). Therefore, even though amino acid sequences of TapA from Aeromonas hydrophila Ah65 and A. salmonicida A450 share $62 \%$ identity, antiserum specific for $A$. hydrophila TapA does not recognize TapA from A. salmonicida (data not shown).

\section{Demonstration that the Aeromonas salmonicida TapD protein has type IV peptidase activity}

Because the Aeromonas salmonicida TapD protein shares a high level of homology (Fig. 2) with members of the type IV prepilin leader peptidase family, whose members are required for type IV pilus biogenesis and/or type II extracellular protein secretion in many Gram-negative pathogens, we predicted that TapD should play a role in one or both of these pathways in $A$. salmonicida. The ability of the $A$. salmonicida TapD protein to act as a prototypical type IV leader peptidase was tested using in vivo and in vitro assays. For these assays, plasmids carrying the Pseudomonas aeruginosa pilD gene (pRBSL.cam), the $A$. salmonicida tapD gene (pCP1227), or the vector (pMMB67EH.cam) alone (Fig. 3A) were transferred into the $P$. aeruginosa pilD mutant strain, PAK 2B18, which is non-piliated because it does not process type IV prepilin (Nunn et al. 1990), and nonhemolytic because of the inability to secrete phospholipase C via the type II pathway (Strom et al. 1991). To determine if TapD is able to functionally complement the $P$. aeruginosa pilD defect, a hemoly- 
A

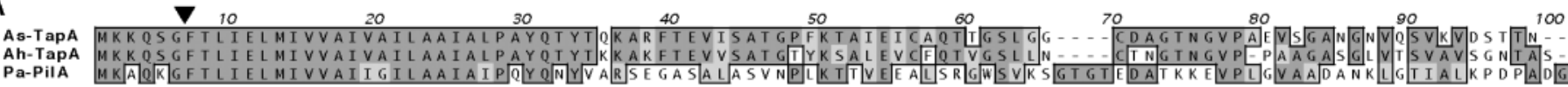

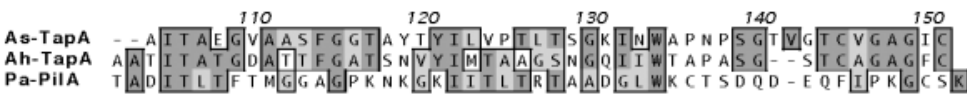

B

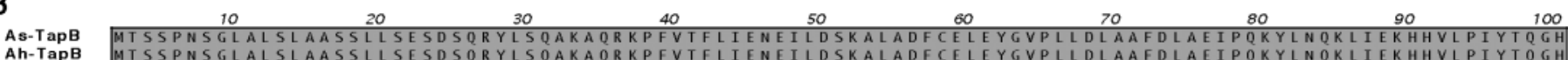

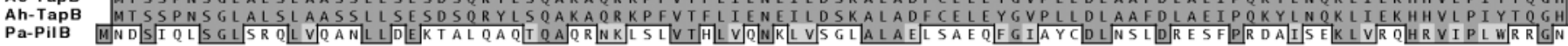

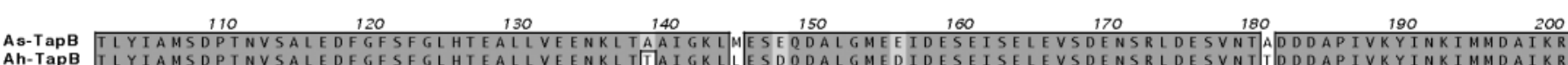

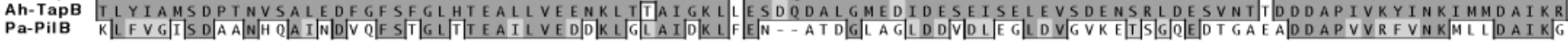

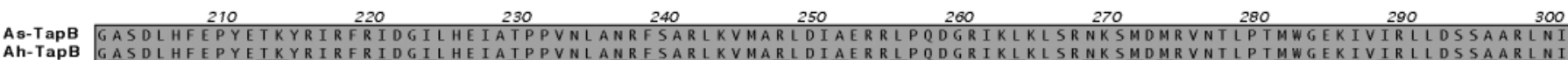

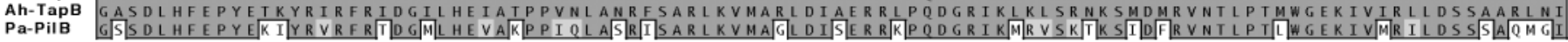

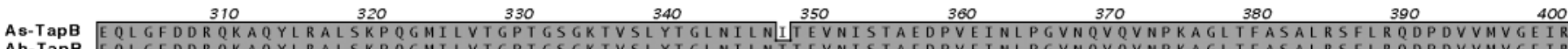

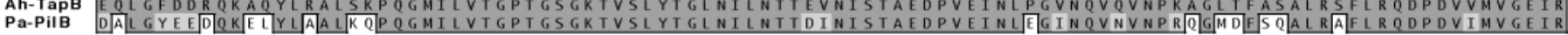

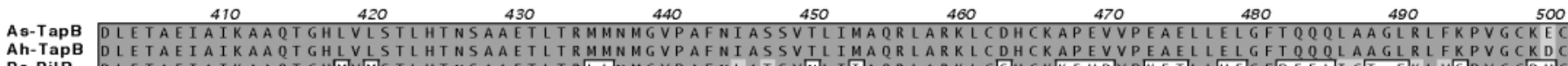

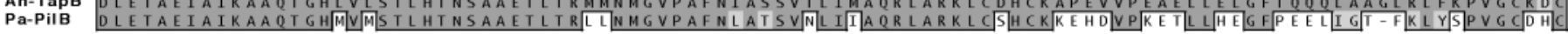

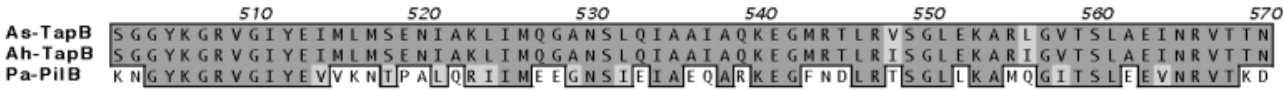

C

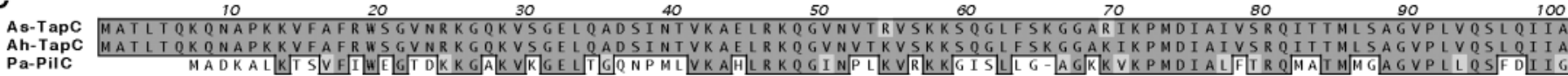

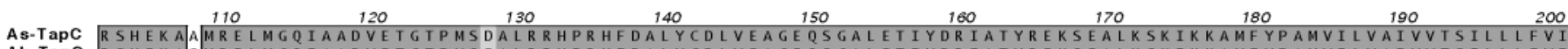
Ah-TaPC RSHEKASSMRLMGOIAADVETGTPMSEALRRHPRHFDALYCDLVEAGEQSGALETIYDRIATYREKSEALKSKIKKAMFYPAMVILVAIVVTSILLLFVI

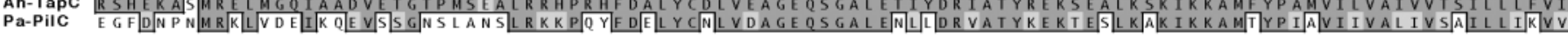

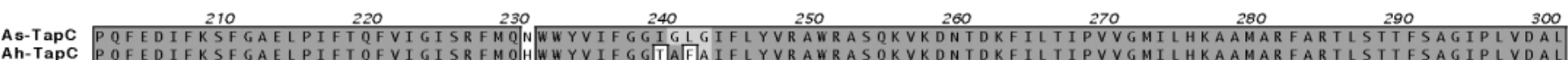

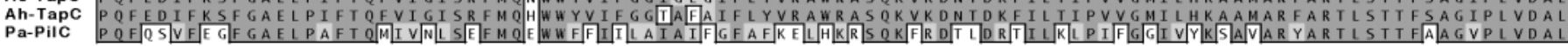

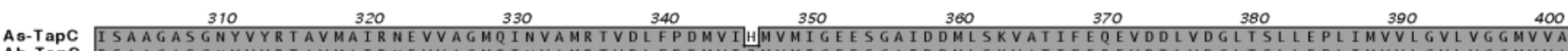

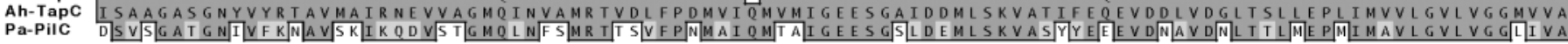

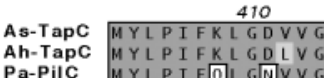

D

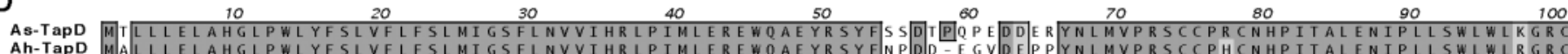

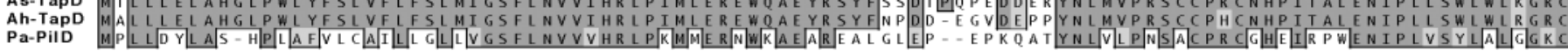

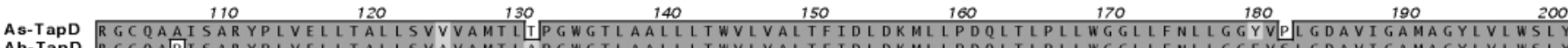

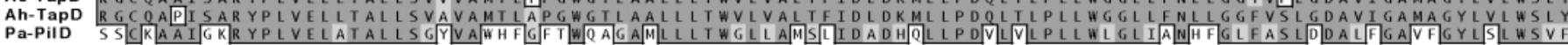

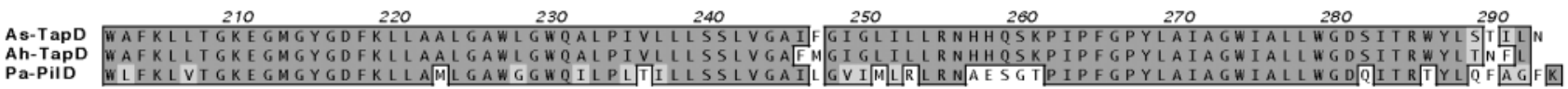

Fig. 2. Amino acid alignments of Tap gene products. ClustalW alignments of Tap gene products from Aeromonas salmonicida (As-Tap) with homologs from A. hydrophila (Ah-Tap) and Pseudomonas aeruginosa (Pa-Pil) are shown. Dark gray shading indicates amino acid identity; light gray shading indicates amino acid similarity. (A) TapA alignment; (B) TapB alignment; (C) TapC alignment; (D) TapD alignment. ( $\mathbf{v}$ ) Predicted cleavage site where the TapA prepilin is cleaved to generate the mature pilin species 
sis assay was performed. The PAK 2B18 transconjugants were patched onto blood-agar plates and as shown in Fig. 3B, overexpressed PilD and TapD both functionally complement the $P$. aeruginosa pilD mutation in vivo. Second, to verify directly that TapD possesses type IV leader peptidase activity, an in vitro cleavage assay (see 'Materials and methods') was performed using $P$. aeruginosa prepilin as substrate. Membrane fractions prepared from the PAK 2B18 transconjugants were incubated with prepilin substrate prepared from a $P$. aeruginosa pilD mutant. As shown in Fig. 3C, the presence of PilD or TapD results in cleavage of the leader peptide from the prepilin substrate generating a mature pilin species. These results show that TapD possesses characteristics typical of type IV prepilin leader peptidases and suggest that $A$. salmonicida has the potential to express type IV pili and/or secrete proteins such as aerolysin via the type II pathway, similar to $A$. hydrophila (Pepe et al. 1996).

\section{A}

1

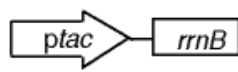

pMMB67HE.cam

2

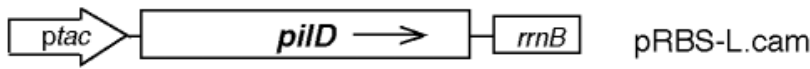

3

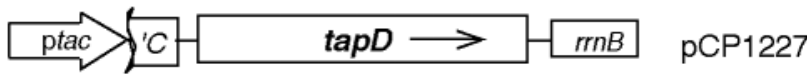

B
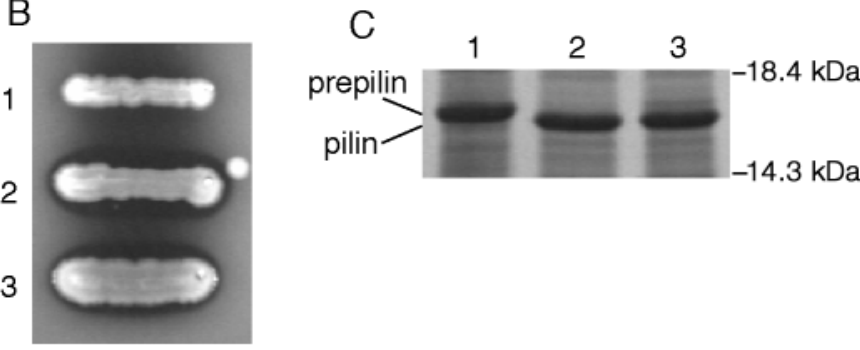

Fig. 3. Type IV leader peptidase activities. (A) Plasmid constructs depicted were used to express Pseudomonas aeruginosa PilD and Aeromonas salmonicida TapD proteins. The inducible promoter, ptac, and part of the Escherichia coli rrnB operon containing the gene for 5S RNA and its 2 transcriptional terminators are shown for each plasmid. The M13mp18 polylinker is located between the tac promoter and the terminators in pMMB67HE.cam. (B) Plasmid constructs in (A) were tested for their ability to functionally complement the pilD defect in the $P$, aeruginosa mutant strain, PAK 2B18, by monitoring their hemolytic activity. (C) Membranes were prepared from $P$. aeruginosa PAK 2B18 containing the plasmids in (A) after overnight growth in the presence of isopropyl thio- $\beta$ D-thiogalactoside (IPTG) . Crude PilD or TapD fractions were incubated with $P$. aeruginosa prepilin. Samples were resolved by $16 \%$ SDS-PAGE and stained with Coomassie brilliant blue R250. Unprocessed prepilin and mature pilin species indicated on the left, molecular weight standards on the right

\section{Construction of a $\operatorname{tap} A$ mutant strain}

Type IV pili have been shown to be important virulence factors in many Gram-negative bacterial species by contributing to adherence and colonization of target host cells (Strom \& Lory 1993). To evaluate the role of type IV Tap pili in Aeromonas salmonicida virulence, a $\operatorname{tap} A$ mutant (A450N1- $\Delta$ A2.3) was constructed in which the wild-type tap $A$ gene was mutated by partial deletion of internal sequences and marked by insertion of a kanamycin resistance gene (see 'Materials and methods'). Because a defect in TapD would be expected to block the biosynthesis of type IV pili, an attempt to construct a tapD mutant was also undertaken. An analogous strategy involving allelic exchange was employed; however, in this case, recovery of a tapD mutant was successful only in the presence of a wild-type copy of tapD (data not shown). Studies are currently underway to determine if tap $D$ is essential for A. salmonicida viability.

To confirm that the $\operatorname{tap} A$ mutation does in fact result in loss of expression from this gene, total RNA from wild type (A450N1) and the $\operatorname{tapA}$ mutant (A450N1$\triangle \mathrm{A} 2.3)$ was subjected to northern blot analysis. A transcript $480 \mathrm{nt}$ in length was detected from A450N1 with a digoxigenin-labeled DNA probe designed to recognize the $\operatorname{tap} A$ transcript (data not shown). This is a reasonable transcript size to expect for a protein 142 amino acids in length. No signal was detected in the lane containing RNA prepared from A450N1- $\Delta$ A2.3 (data not shown).

\section{Examination of $\operatorname{tap} A$ expression}

Examination of both wild-type Aeromonas salmonicida A450N1 and the tapA mutant A450N1- $\Delta$ A2.3 by transmission electron microscopy failed to demonstrate the presence of typical type IV pili on the bacterial surface (data not shown). To confirm TapA expression by $A$. salmonicida, antiserum generated against TapA (see 'Materials and methods') was used for immunoblot analysis on the A450N1, A450N1$\triangle \mathrm{A} 2.3$, and TapA-complemented mutant (A450N1$\Delta \mathrm{A} 2.3 / \mathrm{pCP} 1226)$ strains. Whole-cell lysates of cultures grown in TS broth were subjected to western blot analysis using polyclonal anti-TapA antiserum. The results show that a $\sim 16 \mathrm{kDa}$ protein is recognized by the anti-TapA antiserum in the wild-type strain (Fig. 4, lanes 1 and 3). This band is missing in the lane containing a whole-cell extract from the tapA mutant (Fig. 4, lanes 2 and 4). However, when A450N1- $\Delta$ A2.3 contained a TapA-expressing plasmid (pCP1226) to complement the $\operatorname{tap} A$ defect, the $\sim 16 \mathrm{kDa}$ protein band was again evident (Fig. 4, lane 6). The results 


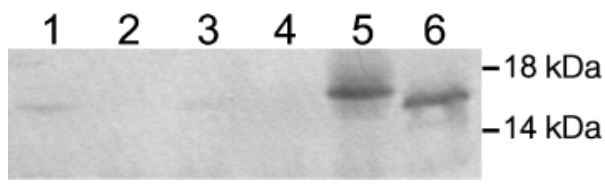

Fig. 4. Expression of TapA in wild-type and tapA mutant strains. Western blot was used to analyze TapA expression from wild type and the tapA mutant strain using polyclonal antiserum directed against TapA-FLAG fusion protein. Lane 1: A450N1 (wild type); lane 2: A450N1- $\Delta$ A2.3 (tapA mutant); lane 3: A450N1/pMMB67HE.cam; lane 4: A450N1$\triangle$ A2.3/pMMB67HE.cam; lane 5: TapA-FLAG fusion protein; lane 6: A450N1- $\Delta$ A2.3/pCP1226 (tapA). Molecular weight standards indicated on the right

obtained from western blot analysis indicate that TapA is expressed at a very low level in wild-type A450N1 under the conditions in which the cells were grown. The level of TapA expression in the presence of pCP1226, the TapA expressing plasmid, is noticeably higher than the wild-type level (Fig. 4, compare lanes 1 and 3 with 6).

\section{In vitro and in vivo effects of a $\operatorname{tap} A$ mutation}

The Aeromonas salmonicida tapA mutant, A450N1$\Delta \mathrm{A} 2.3$, was used to determine if TapA is important for A. salmonicida adherence to cultured fish cell lines. Adherence and colonization of target host cells was tested in vitro on fish cell lines. No significant difference was observed between wild type (A450N1) and the $\operatorname{tap} A$ mutant (A450N1- $\Delta \mathrm{A} 2.3)$ with regard to adherence or invasion of chinook salmon embryo (CHSE)-214 and EPC cells. It should be noted that both strains are invasive on these fish cell lines (data not shown).

The wild-type and tapA mutant strains were also examined for in vivo differences in virulence in rainbow trout. Groups of ninety $5 \mathrm{~g}$ fish were injected with 3 different doses of wild-type Aeromonas salmonicida (A450N1) or the tapA mutant (A450N1$\Delta \mathrm{A} 2.3)$, then held in triplicate tanks. Mortalities were followed for $3 \mathrm{wk}$ and total cumulative mortality is shown in Table 2. Challenge with wild type resulted in a higher mean cumulative mortality compared to the $\operatorname{tap} A$ mutant ( 64 vs $51 \%$ and 11 vs $5 \%$ at the $1: 100$ and 1:1000 doses, respectively). While statistical analysis of the data suggests that this difference is not significant $(p=0.3)$, calculation of the $\mathrm{LD}_{50}$ values (Reed \& Muench 1938) resulted in $5.54 \times 10^{5} \mathrm{cfu} \mathrm{ml}^{-1}$ and $1.37 \times 10^{6} \mathrm{cfu} \mathrm{ml}^{-1}$ for wild type and A450N1$\Delta \mathrm{A} 2.3$, respectively. The 2.5 -fold difference in $\mathrm{LD}_{50}$ values suggests that TapA is involved in virulence of A. salmonicida.
Several fish survived the initial wild-type and $\operatorname{tap} A$ mutant challenges, and it might be expected that the survivors have built up immunity to the bacteria with which they were injected. To determine if a specific immune response is generated against the TapA protein that subsequently offers protection against rechallenge, the surviving fish were subjected to rechallenge with wild-type Aeromonas salmonicida A450N1. After $3 \mathrm{wk}$, the RPS (Amend 1981) for those fish initially challenged with wild type was $70 \%$ compared to $48 \%$ for those initially challenged with the tapA mutant (Table 2). The increase in $\mathrm{LD}_{50}$ in the absence of TapA combined with the evidence that the presence of TapA offers some immunoprotection suggests that TapA is expressed during A. salmonicida infections of rainbow trout and may be important for virulence.

\section{Distribution and expression of $\operatorname{tap} A$ among Aeromonas salmonicida isolates}

To determine whether the $\operatorname{tap} A$ gene is conserved among various isolates of Aeromonas salmonicida from geographically diverse areas, several strains were obtained (Table 1) and their genomic DNA subjected to Southern blot analysis using a Hinc II-Pst I fragment of A. salmonicida A450N1 tapA as a probe (Fig. 1). In all strains tested, as well as A450N1, a 1.8 kb BamHI frag-

Table 2. Aeromonas salmonicida infecting Oncorhynchus mykiss. Rainbow trout challenges conducted in this study. RPS: relative percent survival (Amend 1981)

\begin{tabular}{|c|c|c|c|c|}
\hline & \multicolumn{4}{|c|}{ Cumulative mortality $^{a}$} \\
\hline \multicolumn{5}{|l|}{ Primary challenge $^{a}$} \\
\hline Challenge dose: & 1:100 & 1:1000 & 1:10000 & Tryptone \\
\hline Wild type: & $\begin{array}{c}64 \% \\
(56 / 87)\end{array}$ & $\begin{array}{c}11 \% \\
(10 / 91)\end{array}$ & $\begin{array}{c}2 \% \\
(2 / 90)\end{array}$ & $\begin{array}{c}2 \% \\
(2 / 88)\end{array}$ \\
\hline tapA mutant: & $\begin{array}{c}51 \% \\
(45 / 88)\end{array}$ & $\begin{array}{c}5 \% \\
(4 / 88)\end{array}$ & $\begin{array}{c}2 \% \\
(2 / 90)\end{array}$ & \\
\hline \multicolumn{5}{|c|}{ Survivor challenge $^{b}$} \\
\hline Challenge dose: & $1: 100$ & 1:1000 & 1:10000 & Tryptone \\
\hline Wild type: & $\begin{array}{l}16 \% \\
(5 / 31)\end{array}$ & $\begin{array}{c}36 \% \\
(29 / 81)\end{array}$ & $\begin{array}{c}25 \% \\
(22 / 88)\end{array}$ & $\begin{array}{c}54 \% \\
(46 / 86)\end{array}$ \\
\hline RPS & $70 \%$ & & & \\
\hline tapA mutant: & $\begin{array}{c}28 \% \\
(12 / 43)\end{array}$ & $\begin{array}{c}31 \% \\
(26 / 84)\end{array}$ & $\begin{array}{c}30 \% \\
(26 / 88)\end{array}$ & \\
\hline RPS & $48 \%$ & & & \\
\hline \multicolumn{5}{|c|}{$\begin{array}{l}\text { a Triplicate groups of rainbow trout (mean weight, } 5 \mathrm{~g} \text { ) } \\
\text { were challenged with } 3 \text { different doses of Aeromonas } \\
\text { salmonicida by intraperitoneal injection or with tryptone } \\
\text { (negative control) and monitored } 21 \mathrm{~d} \text { for mortality } \\
\text { b Surviving fish from each dose and treatment above were } \\
\text { rechallenged with a } 1: 100 \text { dilution of a } 1 \%(\mathrm{w} / \mathrm{v}) \text { suspen- } \\
\text { sion of the wild-type A. salmonicida strain }\end{array}$} \\
\hline
\end{tabular}


ment hybridized to the $\operatorname{tap} A$ probe. Western blot analysis on whole-cell extracts from the same strains reacted with polyclonal anti-TapA antiserum prepared against A450N1 TapA, indicating that there is crossreactivity with all the strains examined, although the level of expression under the conditions used here experimentally is low (data not shown).

\section{DISCUSSION}

In this study we describe the cloning and characterization of a 4-gene cluster in Aeromonas salmonicida that encodes elements known to be essential for expression of type IV pili and for extracellular secretion of proteins in many pathogenic Gram-negative bacteria. Type IV pili are a class of pili whose monomolecular subunits share extensive amino acid homology, including a unique leader peptide that must be proteolytically cleaved by a specialized enzyme prior to assembly of the subunits into mature pili (Strom \& Lory 1993). Type IV pili are responsible for a number of important virulence traits of bacteria that possess them, including epithelial cell adherence, biofilm formation, motility, cell-signaling, DNA transformation, and as receptors for bacteriophages (Fussenegger et al. 1997, Kim \& Komano 1997, Kallstrom et al. 1998, Koomey 1998, O'Toole \& Kolter 1998, Semmler et al. 1999, Stone \& Kwaik 1999, Wall \& Kaiser 1999).

The $\operatorname{tap} A$ gene product is a typical type IV pilin of the group A class with a predicted size of 142 amino acids. Group A type IV pilins are initially synthesized as precursors with a short, positively charged leader sequence, a consensus site for cleavage by a type IV prepilin leader peptidase (cleavage site indicated by ' $\mathbf{}$ ' in Fig. 2), and a highly conserved amino terminal domain rich in hydrophobic amino acids. After the leader sequence is cleaved, the newly exposed $\mathrm{N}$-terminal amino acid is $N$-methylated by the same bifunctional type IV leader peptidase prior to assembly (polymerization) of the pilin monomers into pili (Nunn \& Lory 1991, Strom et al. 1993). The translated ORFs of tap $B$ and $\operatorname{tap} C$ have high homology to proteins known to be required for type IV pilus maturation (Strom \& Lory 1993), while the tap $D$ gene product is a member of the type IV prepilin leader peptidases. The Aeromonas salmonicida tapABCD gene cluster is highly homologous to similar gene clusters found in Pseudomonas aeruginosa (Nunn et al. 1990), Vibrio vulnificus (Paranjpye et al. 1998), Vibrio cholerae (termed pil$A B C D$ ) (Fullner \& Mekalanos 1999), and especially $A$. hydrophila (tapABCD) (Pepe et al. 1996). In the $P$. aeruginosa system, where type IV pilus biogenesis is perhaps best understood, the pilABCD gene cluster represents only 4 of approximately 21 total genes, located in 3 genetically unlinked loci on the $P$. aeruginosa chromosome, known to be required for type IV pilus biogenesis (Mattick et al. 1996, Alm \& Mattick 1997). This is likely the case for A. salmonicida as well because sequencing immediately upstream and downstream of the $\operatorname{tap} A B C D$ cluster did not reveal additional pilus biogenesis genes.

Attempts to detect the presence of pili on the surface of Aeromonas salmonicida by electron microscopy combined with immunogold staining have as yet been unsuccessful. However, western immunoblotting demonstrates that TapA is expressed by A. salmonicida in vitro. While this is not proof of bacterial piliation, there are no known instances where a type IV pilin protein is expressed and not assembled into functional pili. Western blots do demonstrate that the amount of pilin protein expressed by A. salmonicida appears to be at a low level, and indeed similar low levels of expression under laboratory culture conditions have been demonstrated for the TapA type IV pili of $A$. hydrophila (Pepe et al. 1996, Kirov et al. 2000) and Vibrio vulnificus (R. N. Paranjpye \& M. S. Strom unpubl.). However, low pilin expression could simply reflect a lack of appropriate environmental cues in the in vitro growth conditions used because it is known that expression of type IV pilins are under transcriptional control by members of protein-phosphorylating 2-component regulatory systems and the alternative RpoN sigma factor of RNA polymerase (Ishimoto \& Lory 1989, Jin et al. 1994). A consensus RpoN-dependent promoter is located upstream of the putative ribosome binding site of $A$. salmonicida tapA.

Assigning a possible functional role for the putative Aeromonas salmonicida type IV pili has likewise proven difficult. Type IV pili mediate adherence of Gramnegative pathogens to various types of epithelial, plant, and fungal cells and it is thought that adherence is an important initial colonization, and thus virulence, factor (Dorr et al. 1998, O'Toole \& Kolter 1998). However, adherence assays using fish cell lines such as the salmonid-derived CHSE-214 or the cyprinid EPC line revealed no differences in adherence or in subsequent intracellular invasion between the wild-type and $\operatorname{tap} A$ mutant strains. Comparison of the cumulative mortality and $\mathrm{LD}_{50}$ values obtained with the wild-type and $\operatorname{tap} A$ mutant strains after an in vivo injection challenge of rainbow trout did reveal a small but reproducible difference. On average, the $\mathrm{LD}_{50}$ of the $\operatorname{tap} A$ mutant was approximately 2.5 -fold higher than the wild-type strain, suggesting that expression of TapA is an important factor in A. salmonicida virulence.

The results obtained with wild-type Aeromonas salmonicida rechallenge of fish initially challenged with a sublethal dose of the wild-type or tapA mutant strains supports the hypothesis that TapA is expressed in vivo. 
The presence of TapA on A. salmonicida allowed the wild-type strain to generate a higher level of protection against rechallenge with wild type as compared to the $\operatorname{tap} A$ mutant strain in the survivors challenged at the higher dose. Type IV pili are potent immunogens that stimulate protective immunity in many mammalian infections, including humans infected with Neisseria gonorrhoeae or Vibrio cholerae (Strom \& Lory 1993), and in fact, form the basis for vaccines against the animal pathogens Moraxella bovis and Dichelobacter nodosus (Stewart et al. 1985, Lepper 1988). More recently, the toxin co-regulated pilus (TCP), a group B type IV pilin in $V$. cholerae (Herrington et al. 1988), has been shown to have promise as a protective cholera vaccine (Wu et al. 2001). These results suggest that the TapA type IV pilin could form the basis for a protein subunit vaccine against $A$. salmonicida. For this idea to have merit Tap pili must be widespread in A. salmonicida and there must be cross-reactivity in the immune response generated against a variety of $A$. salmonicida strains. While it is true in many cases that type IV pili are antigenically heterogeneous in some bacterial species, they are not in others (Strom \& Lory 1993). Data presented here suggest that A. salmonicida TapA pili are more homogeneous, as polyclonal antiserum raised against $A$. salmonicida A450N1 TapA crossreacts with an identically sized protein from a variety of A. salmonicida strains.

Type IV prepilin leader peptidases, of which TapD is a member, process prepilins as well as components of the type II secretion system apparatus (pre-pseudopilins) (Lory \& Strom 1997, Strom \& Lory 2001). Many virulence factors are secreted by type II secretion pathways, including Pseudomonas aeruginosa exotoxin A (Strom et al. 1991), Aeromonas hydrophila aerolysin (Pepe et al. 1996), and Vibrio cholerae cholera toxin (Marsh \& Taylor 1998). In A. salmonicida these include the precursor form of aerolysin (Burr et al. 2001), the GCAT toxin (glycerophospholipid:cholesterol acyltransferase) (Brumlik et al. 1997), the paracrystalline surface array protein (A-layer or S-layer) (Trust 1993, Noonan \& Trust 1995, Thomas \& Trust 1995), and possibly the A. salmonicida hemolysins (ASH) and a serine protease. The functional characterization of TapD, demonstrating that it can efficiently complement a $P$. aeruginosa PilD defect by processing prepilin and prepseudopilins to restore pilus expression and type II secretion in $P$. aeruginosa, supports the conclusion that TapD is a type IV prepilin leader peptidase.

Due to its role in both type IV pilus biogenesis and type II secretion, a mutation in $\operatorname{tap} D$ is likely to have a pleiotropic effect on the Aeromonas salmonicida phenotype. Such a mutant would be especially interesting for the study of $A$. salmonicida because defined mutations in single toxin genes often do not result in atten- uated virulence (Vipond et al. 1998). We observed a pleiotropic secretion defect in an A. hydrophila tapD mutant (Pepe et al. 1996), but we have not been able to construct such a mutation in A. salmonicida in the absence of TapD expression in trans, which suggests that TapD is essential for A. salmonicida viability. This would be unique among the many Gram-negative pathogens in which expression of the type IV leader peptidase gene has been abolished without concomitant loss of viability (Nunn et al. 1990, Pepe et al. 1996, Paranjpye et al. 1998, Fullner \& Mekalanos 1999). We are currently attempting other strategies to mutate or delete the $\operatorname{tap} D$ gene in order to verify this finding.

This is the first report describing expression of a protein with homology to type IV pilins in Aeromonas salmonicida. The demonstration of a role in virulence along with evidence that the protein is involved in rainbow trout immune response against infection with the pathogen warrants further investigation of the protein's actual function in pathogenesis and as a potential vaccine candidate. In ongoing and future studies we hope to answer both questions.

Acknowledgements. We would like to thank the following individuals for their assistance in various aspects of this work: Bill Batts for DNA sequencing, David Powell and Debra Milton for Aeromonas salmonicida strains, Jerry Jones and Aaron Werghall for their assistance with the in vivo studies, Karl Shearer for statistical analyses, and Linda Rhodes for critically reading the manuscript. This research was supported by a grant from the National Research Initiative Grants Program (contract number 98-35204-6979) administered by the United States Department of Agriculture. Additional support was provided by the National Marine Fisheries Service, National Oceanic and Atmospheric Administration, United States Department of Commerce.

\section{LITERATURE CITED}

Alm RA, Mattick JS (1997) Genes involved in the biogenesis and function of type-4 fimbriae in Pseudomonas aeruginosa. Gene 192:89-98

Altschul SF, Madden TL, Schäffer AA, Zhang J, Zhang Z, Miller W, Lipman DJ (1997) Gapped BLAST and PSI-BLAST: a new generation of protein database search programs. Nucleic Acids Res 25:3389-3402

Amend DF (1981) Potency testing of fish vaccines. Dev Biol Stand 49:447-454

Barnett TC, Kirov SM (1999) The type IV Aeromonas pilus (Tap) gene cluster is widely conserved in Aeromonas species. Microb Pathog 26:77-84

Barnett TC, Kirov SM, Strom MS, Sanderson K (1997) Aeromonas spp. possess at least two distinct type IV pilus families. Microb Pathog 23:241-247

Birnboim HC, Doly J (1979) A rapid alkaline extraction procedure for screening recombinant plasmid DNA. Nucleic Acids Res 7:1513-1523

Brumlik MJ, van der Goot FG, Wong KR, Buckley JT (1997) The disulfide bond in the Aeromonas hydrophila lipase/ 
acyltransferase stabilizes the structure but is not required for secretion or activity. J Bacteriol 179:3116-3121

Buckley JT, Halasa LN, MacIntyre S (1982) Purification and partial characterization of a bacterial phospholipid: cholesterol acyltransferase. J Biol Chem 257:3320-3325

Burr SE, Diep DB, Buckley JT (2001) Type II secretion by Aeromonas salmonicida: evidence for two periplasmic pools of proaerolysin. J Bacteriol 183:5956-5963

Dorr J, Hurek T, Reinhold-Hurek B (1998) Type IV pili are involved in plant-microbe and fungus-microbe interactions. Mol Microbiol 30:7-17

Ellis AE (1997) The extracellular toxins of Aeromonas salmonicida subsp. salmonicida. In: Bemoth E, Ellis AE, Midtlyng PJ, Olivier G, Smith P (eds) Furunculosis. Academic Press, San Diego, p 248-268

Fullner KJ, Mekalanos JJ (1999) Genetic characterization of a new type IV-A pilus gene cluster found in both classical and El Tor biotypes of Vibrio cholerae. Infect Immun 67:1393-1404

Fussenegger M, Rudel T, Barten R, Ryll R, Meyer TF (1997) Transformation competence and type-4 pilus biogenesis in Neisseria gonorrhoeae-a review. Gene 192:125-134

Gay P, Le Coq D, Steinmetz M, Berkelman T, Kado CI (1985) Positive selection procedure for entrapment of insertion sequence elements in Gram-negative bacteria. J Bacteriol 164:918-921

Herrington DA, Hall RH, Losonsky G, Mekalanos JJ, Taylor RK, Levine MM (1988) Toxin, toxin-coregulated pili, and the toxR regulon are essential for Vibrio cholerae pathogenesis in humans. J Exp Med 168:1487-1492

Hirono I, Aoki T (1993) Cloning and characterization of three hemolysin genes from Aeromonas salmonicida. Microb Pathog 15:269-282

Ishiguro EE, Kay WW, Ainsworth T, Chamberlain JB, Austen RA, Buckley JT, Trust TJ (1981) Loss of virulence during culture of Aeromonas salmonicida at high temperature. J Bacteriol 148:333-340

Ishimoto KS, Lory S (1989) Formation of pilin in Pseudomonas aeruginosa requires the alternative sigma factor $(\mathrm{RpoN})$ of RNA polymerase. Proc Natl Acad Sci USA 86:1954-1957

Jiang B, Howard SP (1992) The Aeromonas hydrophila exeE gene, required both for protein secretion and normal outer membrane biogenesis, is a member of a general secretion pathway. Mol Microbiol 6:1351-1361

Jin S, Ishimoto KS, Lory S (1994) PilR, a transcriptional regulator of piliation in Pseudomonas aeruginosa, binds to a cis-acting sequence upstream of the pilin gene promoter. Mol Microbiol 14:1049-1057

Kallstrom H, Islam MS, Berggren PO, Jonsson AB (1998) Cell signaling by the type IV pili of pathogenic Neisseria. J Biol Chem 273:21777-21782

Kim SR, Komano T (1997) The plasmid R64 thin pilus identified as a type IV pilus. J Bacteriol 179:3594-3603

Kirov SM, Barnett TC, Pepe CM, Strom MS, Albert MJ (2000) Investigation of the role of type IV Aeromonas pilus (Tap) in the pathogenesis of Aeromonas gastrointestinal infection. Infect Immun 68:4040-4048

Koomey M (1998) Competence for natural transformation in Neisseria gonorrhoeae: a model system for studies of horizontal gene transfer. APMIS Suppl 84:56-61

Laemmli UK (1970) Cleavage of structural proteins during the assembly of the head of bacteriophage T4. Nature 227: 680-685

Lepper AW (1988) Vaccination against infectious bovine keratoconjunctivitis: protective efficacy and antibody response induced by pili of homologous and heterologous strains of Moraxella bovis. Aust Vet J 65:310-316
Lory S, Strom MS (1997) Structure-function relationship of type-IV prepilin peptidase of Pseudomonas aeruginosaa review. Gene 192:117-121

Manoil C, Beckwith J (1986) A genetic approach to analysing membrane protein topology. Science 233:1403-1408

Marsh JW, Taylor RK (1998) Identification of the Vibrio cholerae type 4 prepilin peptidase required for cholera toxin secretion and pilus formation. Mol Microbiol 29: 1481-1492

Mattick JS, Whitchurch CB, Alm RA (1996) The molecular genetics of type-4 fimbriae in Pseudomonas aeruginosaa review. Gene 179:147-155

Merino S, Aguilar A, Rubires X, Simon-Pujol D, Congregado F, Tomas JM (1996) The role of the capsular polysaccharide of Aeromonas salmonicida in the adherence and invasion of fish cell lines. FEMS Microbiol Lett 142:185-189

Miller VL, Mekalanos JJ (1988) A novel suicide vector and its use in construction of insertion mutations: osmoregulation of outer membrane proteins and virulence determinants in Vibrio cholerae requires toxR. J Bacteriol 170:2575-2583

Noonan B, Trust TJ (1995) Molecular analysis of an A-protein secretion mutant of Aeromonas salmonicida reveals a surface layer-specific protein secretion pathway. J Mol Biol 248:316-327

Nunn D (1999) Bacterial type II protein export and pilus biogenesis: more than just homologies? Trends Cell Biol 9:402-408

Nunn DN, Lory S (1991) Product of the Pseudomonas aeruginosa gene pilD is a prepilin leader peptidase. Proc Natl Acad Sci USA 88:3281-3285

Nunn DN, Bergman S, Lory S (1990) Products of three accessory genes, pilB, pilC, and pilD, are required for biogenesis of Pseudomonas aeruginosa pili. J Bacteriol 172: 2911-2919

O'Toole GA, Kolter R (1998) Flagellar and twitching motility are necessary for Pseudomonas aeruginosa biofilm development. Mol Microbiol 30:295-304

Paranjpye RN, Lara JC, Pepe JC, Pepe CM, Strom MS (1998) The type IV leader peptidase/N-methyltransferase of Vibrio vulnificus controls factors required for adherence to HEp-2 cells and virulence in iron-overloaded mice. Infect Immun 66:5659-5668

Pepe CM, Eklund MW, Strom MS (1996) Cloning of an Aeromonas hydrophila type IV pilus biogenesis gene cluster: complementation of pilus assembly functions and characterization of a type IV leader peptidase/N-methyltransferase required for extracellular protein secretion. Mol Microbiol 19:857-869

Pugsley AP (1993) The complete general secretory pathway in Gram-negative bacteria. Microbiol Rev 57:50-108

Pugsley AP, Francetic O, Possot OM, Sauvonnet N, Hardie KR (1997) Recent progress and future directions in studies of the main terminal branch of the general secretory pathway in Gram-negative bacteria - a review. Gene 192: 13-19

Quandt J, Hynes MF (1993) Versatile suicide vectors which allow direct selection for gene replacement in Gramnegative bacteria. Gene 127:15-21

Reed LJ, Muench H (1938) A simple method of estimating the $50 \%$ endpoints. Am J Hyg 27:493-497

Sakai DK (1985) Loss of virulence in a protease-deficient mutant of Aeromonas salmonicida. Infect Immun 48: 146-152

Sambrook J, Fritsch EF, Maniatis T (1989) Molecular cloning: a laboratory manual. Cold Spring Harbor Laboratory Press, Cold Spring Harbor, NY

Semmler AB, Whitchurch CB, Mattick JS (1999) A re-exami- 
nation of twitching motility in Pseudomonas aeruginosa. Microbiology 145:2863-2873

Simon R, Priefer U, Pühler A (1983) A broad host range mobilization system for in vivo genetic engineering: transposon mutagenesis in Gram-negative bacteria. Bio/Technology 1:784-791

Stewart DJ, Clark BL, Peterson JE, Emery DL, Smith EF, Griffiths DA, O'Donnell IJ (1985) The protection given by pilus and whole cell vaccines of Bacteroides nodosus strain 198 against ovine foot-rot induced by strains of different serogroups. Aust Vet J 62:153-159

Stone BJ, Kwaik YA (1999) Natural competence for DNA transformation by Legionella pneumophila and its association with expression of type IV pili. J Bacteriol 181: 1395-1402

Strom MS, Lory S (1986) Cloning and expression of the pilin gene of Pseudomonas aeruginosa PAK in Escherichia coli. J Bacteriol 165:367-372

Strom MS, Lory S (1993) Structure-function and biogenesis of the type IV pili. Annu Rev Microbiol 47:565-596

Strom MS, Lory S (2001) Type IV prepilin leader peptidases. In: Dalbey RE, Sigman DS (eds) The enzymes, Vol XXII. Academic Press, San Diego, p 127-159

Strom MS, Nunn D, Lory S (1991) Multiple roles of the pilus biogenesis protein PilD: involvement of PilD in excretion of enzymes from Pseudomonas aeruginosa. J Bacteriol 173:1175-1180

Strom MS, Nunn DN, Lory S (1993) A single bifunctional enzyme, PilD, catalyzes cleavage and $N$-methylation of proteins belonging to the type IV pilin family. Proc Natl Acad Sci USA 90:2404-2408

Studier FW, Moffatt BA (1986) Use of bacteriophage T7 RNA

Editorial responsibility: Carey Cunningham,

Aberdeen, Scotland, UK polymerase to direct selective high-level expression of cloned genes. J Mol Biol 189:113-130

Thomas SR, Trust TJ (1995) A specific PulD homolog is required for the secretion of paracrystalline surface array subunits in Aeromonas hydrophila. J Bacteriol 177: 3932-3939

Towbin H, Staehelin T, Gordon J (1979) Electrophoretic transfer of proteins from polyacrylamide gels to nitrocellulose sheets: procedure and some applications. Proc Natl Acad Sci USA 76:4350-4354

Trust TJ (1993) Molecular, structural and functional properties of Aeromonas S-layers. In: Beveridge TJ, Koval SF (eds) Advances in bacterial paracrystalline surface layers. Plenum Publishing Corporation, New York, p 159-171

Turner LR, Lara JC, Nunn DN, Lory S (1993) Mutations in the consensus ATP-binding sites of XcpR and PilB eliminate extracellular protein secretion and pilus biogenesis in Pseudomonas aeruginosa. J Bacteriol 175:4962-4969

Vipond R, Bricknell IR, Durant E, Bowden TJ, Ellis AE, Smith M, MacIntyre S (1998) Defined deletion mutants demonstrate that the major secreted toxins are not essential for the virulence of Aeromonas salmonicida. Infect Immun 66: 1990-1998

Wall D, Kaiser D (1999) Type IV pili and cell motility. Mol Microbiol 32:1-10

Wu JY, Wade WF, Taylor RK (2001) Evaluation of cholera vaccines formulated with toxin-coregulated pilin peptide plus polymer adjuvant in mice. Infect Immun 69: 7695-7702

Yim HH, Villarejo M (1992) osm Y, a new hyperosmotically inducible gene, encodes a periplasmic protein in Escherichia coli. J Bacteriol 174:3637-3644

Submitted: October 15, 2001; Accepted: February 18, 2002 Proofs received from author(s): July 18, 2002 